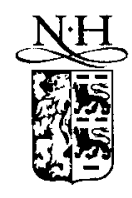

ELSEVIER

\title{
Finite element formulation for non-smooth oscillations of an elastic beam with a friction device
}

\author{
P. Vielsack*, H. Kammerer \\ Universitat Karlsruhe, Institut fur Mechanik, Kaiserstr. 12, Postfach 69 80, 76128 Karlsruhe, Germany
}

Received 18 May 1998

\begin{abstract}
Calculated are forced oscillations of a clamped elastic beam with a friction device at its free end. Considering partial states, namely sticking and sliding, and applying FEM leads to a discrete non-smooth dynamic problem. Stationary oscillations are of interest, depending on the number of finite elements and on different types of friction laws. Due to the linearity of partial states of motion, the numerical integration is reduced to the calculation of switching times which separate the sequence of partial states. Appropriate chosen internal damping diminishes the numerical effort considerably. (C) 1999 Elsevier Science S.A. All rights reserved.
\end{abstract}

\section{Introduction}

The dissipation of mechanical energy in vibratory systems can be realized in several ways. One method is to directly use dry friction, with a distinction between continuous friction [1] and local friction in friction devices. The latter situation is being investigated in the following. In practice, friction devices are mainly employed in one-dimensional continua, e.g. bars and beams [2].

Dry friction naturally leads to non-smooth dynamic systems with strong nonlinearity. The operator $\boldsymbol{F}$ in a non-smooth system $\dot{\boldsymbol{X}}=\boldsymbol{F}(\boldsymbol{X}, t)$ is discontinuous. The range of definition of $\boldsymbol{F}$ can be split in a set $\mathcal{M}$ with $k$ smooth parts $\boldsymbol{F}=\cup_{j=1}^{k} \boldsymbol{f}^{(j)} ; \boldsymbol{f}^{(j)} \in \mathcal{M}$ [3]. The total solution $\boldsymbol{X}$ is constructed by a sequence of partial solutions $\boldsymbol{X}^{(i)} ; i=1,2, \ldots$, which depend on the time history. The transition from $\boldsymbol{X}^{(i-1)}$ to $\boldsymbol{X}^{(i)}$ occurs at a certain switching time $t_{i-1}$ which separates consecutive states. The sequence of switching times $t_{0}<t_{1}<\cdots t_{i-1}<t_{i}<$ $\cdots$ is a priori unknown. At $t_{i-1}$ the actual partial operator valid for $t>t_{i-1}$ must be chosen out of a set $\mathcal{M}$. For this purpose, switching decisions must be defined. In addition, the known partial solution $\boldsymbol{X}^{(i-1)}$ at $t_{i-1}$ gives the initial conditions for the following partial solution $\boldsymbol{X}^{(i)}$. Its range of definition is controlled by switching conditions which allow to calculate the following switching time $t_{i}$.

Comprehensive literature on this subject has been made available recently [4], which, however, is focused on rigid-body dynamics. In contrast, contact problems in continuum mechanics lead to systems with finite numbers of degrees of freedom after spatial discretization by the finite element method. The main point in question is to find a realistic, but comprehensible, typical mechanical system, to describe its dynamic properties and to formulate the algorithm to calculate its oscillation. Here, the question arises how the spatial discretization of the continuum by finite elements affects the necessary discretization in time for integration of the motion.

The mathematical problem of non-smooth dynamics can be split into two tasks, namely the time integration of successive smooth systems arising from the FE-formulation and the determination of the switching times. Both tasks can be performed numerically with limited accuracy [5]. The influence of both kinds of numerical errors

\footnotetext{
* Corresponding author. E-mail: mechanik@bau-verm.uni-karlsruhe.de
} 
cannot be separated. As will be shown later, the partial smooth systems are linear. Their general solutions can be given explicitely by well-known methods of linear vibration theory even for large numbers of finite elements. Taking advantage of this possibility, the numerical task is reduced to the calculation of the switching times as roots of algebraic equations. This semianalytical procedure allows to focus the investigation on the distinct properties of non-smooth systems especially on the existence of switching conditions and decisions. Moreover, a modal decomposition of the linear partial motions allows a discussion about the influence of higher vibrational modes on the calculation of the motion.

\section{Mechanical system and force laws}

Consider an Euler-Bernoulli beam fixed at one end, which has at its free end a support with dry friction. Displacements in the transverse direction are possible. The beam is excited in its middle by a harmonic force

$$
F=F_{0} \sin \Omega t .
$$

By means of the FE method, the continuous problem is discretized according to the known procedure [5] by $N$ beam elements $(N \geqslant 2$, even) (see Fig. 1).

At each of the $N$ nodes we introduce two degrees of freedom with regard to rotation and displacement. The displacement of the friction device is treated separately and denominated by $y$. The other ones are combined to one vector $\boldsymbol{x}$. Hence, the global coordinate vector of the dimension $2 N$ reads

$$
\boldsymbol{q}^{\mathrm{T}}=[\{\boldsymbol{x}\}, y] .
$$

Due to the friction support, the boundary conditions of the beam are not unique. During the oscillation intermittant constraints must be considered. Three possible partial motions may occur: 'sticking' with vanishing velocity $\dot{y} \equiv 0$, 'sliding' with a velocity $\dot{y}>0$ or 'sliding' with $\dot{y}<0$.

In the state 'sticking', the support is fixed but shifted against the tensionless straight position of the beam by a displacement $y_{0}$ constant in time. The value of $y_{0}$ follows from the previous history of the motion. This partial state has $2 N-1$ degrees of freedom with the coordinate vector $[\boldsymbol{x}]$. The appropriate contact force $R(t)$ is a passive force which can be calculated from the known motion in this state.

In both sliding states, the contact force is an active force with a given law of force

$$
R(\dot{y})=R_{0} \operatorname{sgn} \dot{y}+d \dot{y} ; \quad|\dot{y}|>0 .
$$

The parameter $R_{0}$ is an adjustable threshold value resulting from a constant compression due to prestressing the friction device and a friction coefficient. The value $d$ rules the slope of the characteristic of the friction law for $|\dot{y}|>0$. In this connection it has to be pointed out that the term $d \dot{y}$ in (3) must not be mistaken as an internal damping of the beam. In the state 'sticking' described before, this term does not exist which means that it is clearly associated with a state 'sliding'. A value $d=0$ in (3) corresponds to Coulomb's law. Characteristics with $d>0$ and $d<0$ yield increasing and decreasing friction forces with increasing values of velocity (Fig. 2). In both states 'sliding' the beam has no constraint at its free end. These partial states are therefore characterized by $2 N$ degrees of freedom with the coordinate vector $[\{\boldsymbol{x}\}, y]$ according to (2).

In the time domain, the non-smooth dynamical behaviour of the beam with a friction device consists of a sequence of smooth partial motions. Each of these partial motions is linear and can be conceived by the known

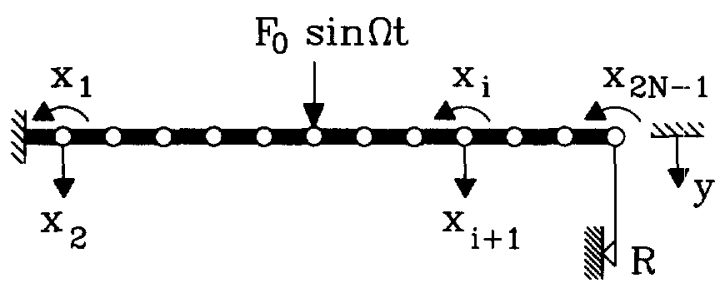

Fig. 1. Discretized beam. 


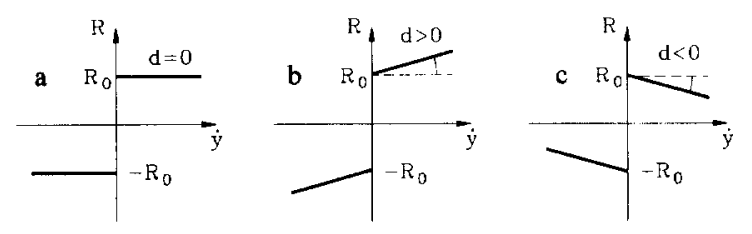

Fig. 2. Force laws: (a) Coulomb; (b) increasing; (c) decreasing.

methods of the theory of vibrations of systems with finite degrees of freedom. The nonlinearity of the total oscillation problem is therefore based on two fundamental unknown circumstances:

- the kind of succession of the smooth partial motions (sequence of partial states),

- the time of transition from one smooth motion to the following one (switching times between partial states).

Both facts result from the oscillation history. If a smooth motion at the time $t_{0}$ is assumed to be known, its behaviour with increasing time $t>t_{0}$ is decisive for its end and for the type of the following smooth motion.

\section{Motion equations and succession of partial states}

The global system matrices for a discretization by $N$ elements are obtained by the finite element method [6]. In accordance with the representation of the coordinate vector (2), these matrices are composed of submatrices. Mass $(\boldsymbol{M})$, damping $(\boldsymbol{D})$ and stiffness $(\boldsymbol{K})$ have the following structure

$$
\boldsymbol{M}=\left[\begin{array}{cc}
\left\{\boldsymbol{M}_{1,1}\right\} & \left\{\boldsymbol{M}_{1,2 N}\right\} \\
\left\{\boldsymbol{M}_{1,2 N}^{\mathrm{T}}\right\} & m_{2 N, 2 N}
\end{array}\right], \quad \boldsymbol{D}=\left[\begin{array}{cc}
\left\{\boldsymbol{D}_{1,1}\right\} & \left\{\boldsymbol{D}_{1,2 N}\right\} \\
\left\{\boldsymbol{D}_{1,2 N}^{\mathrm{T}}\right\} & d_{2 N, 2 N}
\end{array}\right], \quad \boldsymbol{K}=\left[\begin{array}{cc}
\left\{\boldsymbol{K}_{1,1}\right\} & \left\{\boldsymbol{K}_{1,2 N}\right\} \\
\left\{\boldsymbol{K}_{1,2 N}^{\mathrm{T}}\right\} & k_{2 N, 2 N}
\end{array}\right]
$$

The vector of the excitation force

$$
\boldsymbol{F}^{\mathrm{T}}=[\{\mathbf{0}\}, F(t),\{\boldsymbol{0}\}]=\left[\left\{\boldsymbol{F}_{1}(t)\right\}, 0\right]
$$

has a non-vanishing entry for the force excitation (1) at the middle node only. According to the representation of the coordinate vector (2), the entry at the friction device is treated seperately. The same applies to the contact force vector

$$
\boldsymbol{R}^{T}=[\{\mathbf{0}\}, R] .
$$

\subsection{State 'sticking'}

Assume $t=t_{i-1}$ to be a known switching time, at which the partial motion starts where the friction device sticks. The previous history at the time $t_{i-1}-0$ yields all coordinates $\boldsymbol{x}\left(t_{i-1}-0\right), y\left(t_{i-1}-0\right)=y_{0}$ and velocities $\dot{x}\left(t_{i-1}-0\right), \dot{y}\left(t_{i-1}-0\right)=0$. They form $2(2 N-1)$ initial conditions

$$
\begin{aligned}
& \boldsymbol{x}\left(t_{i-1}+0\right)=\boldsymbol{x}\left(t_{i-1}-0\right), \\
& \dot{x}\left(t_{i-1}+0\right)=\dot{x}\left(t_{i-1}-0\right)
\end{aligned}
$$

for the $2 N-1$ linear equations of motion

$$
M_{1,1} \ddot{x}+D_{1,1} \dot{x}+K_{1,1} x=F_{1}-y_{0} K_{1,2 N} .
$$

The friction device itself is governed by $y(t) \equiv y_{0}$ and $\dot{y}(t) \equiv 0$ for $t>t_{i-1}$. The motion of the beam occurs around a curved shape of the beam given by the known displacement $y_{0}$. The contact force (passive force) results from an algebraic equation

$$
R(t)=\boldsymbol{M}_{1,2 N}^{\mathrm{T}} \ddot{\boldsymbol{x}}+\boldsymbol{D}_{1,2 N}^{\mathrm{T}} \dot{\boldsymbol{x}}+\boldsymbol{K}_{1,2 N}^{\mathrm{T}} \boldsymbol{x}+y_{0} k_{2 N, 2 N} .
$$

The partial state 'sticking' exists in a time interval $t_{i-1}<t<t_{i}$. The unknown switching time $t_{i}$ characterizes the change to a new state. Sticking is finished as soon as the value of the contact force reaches the threshold value $R_{0}$ in Eq. (3). Thus, the switching condition 


$$
|R(t)|=R_{0}
$$

corresponds to the search for the first root of $(10)$ in $\left(t_{i-1}, t[\right.$. Sticking is followed by sliding. The switching decision

$$
\operatorname{sgn}\left(\dot{y}\left(t_{i}+0\right)\right)=\operatorname{sgn}\left(R\left(t_{i}-0\right)\right)
$$

rules the direction of sliding. At the switching point, the contact force is continuous, but not continuously differentiable.

\subsection{States 'sliding'}

Assume $t=t_{i-1}$ to be a known switching time, at which a partial state 'sliding' in a known direction starts. The previous history yields all coordinates $q\left(t_{i-1}-0\right)$ and velocities $\dot{q}\left(t_{i-1}-0\right)$ at the time $t_{i-1}-0$. They form $2 \times 2 N$ initial conditions

$$
\begin{aligned}
& \boldsymbol{q}\left(t_{i-1}+0\right)=\boldsymbol{q}\left(t_{i-1}-0\right), \\
& \boldsymbol{q}\left(t_{i-1}+0\right)=\boldsymbol{q}\left(t_{i-1}-0\right)
\end{aligned}
$$

for the $2 N$ equations of motion

$$
\boldsymbol{M} \ddot{\boldsymbol{q}}+\boldsymbol{D} \boldsymbol{q}+\boldsymbol{K} \boldsymbol{q}=\boldsymbol{F}-\boldsymbol{R}(\dot{y}) .
$$

The contact force $\boldsymbol{R}$ is an active friction force given by the force law (3). Because its direction is known from the history, Eq. (13) is linear. Both states 'sliding' are described by the same equation (13). The only difference between both states is the sign of the constant $R_{0}$, which can be seen from Egs. (6) and (3). Both partial states 'sliding' exist in a time range $t_{i-1}<t<t_{i}$. The unknown switching time $t_{i}$ characterizes the transition to the new state. Sliding is finished when the velocity $y$ becomes zero. The search for the first root of (14) in the open interval $\left(t_{i-1}, t[\right.$ thus corresponds to the switching condition

$$
\dot{y}(t)=0 \text {. }
$$

For the new state starting at $t_{i}+0$ there are two possibilities: first, 'sticking' is presupposed. Then the contact force can be calculated from (9) as the passive force $R\left(t_{i}+0\right)$. If the switching condition

$$
\left|R\left(t_{i}+0\right)\right|<R_{0}
$$

is met, 'sticking' really exists in the open interval $\left(t_{i-1}, t[\right.$. If, on the other hand,

$$
\left|R\left(t_{i}+0\right)\right|>R_{0}
$$

we have an immediate reversal of motion to opposite sliding

$$
\operatorname{sgn}\left(\dot{y}\left(t_{i}+0\right)\right)=-\operatorname{sgn}\left(\dot{y}\left(t_{i}-0\right)\right) .
$$

At the end of each sliding process, the contact force is discontinuous.

Both smooth linear initial value problems (12), (13) or, respectively (7), (8) are treated using classic methods of the linear theory of vibration. Assuming internal damping

$$
D=\alpha M+\beta K
$$

of the beam in the form of Rayleigh damping, analytical solutions for all partial problems result by a modal decomposition. In the case 'sticking' $2 N-1$ natural frequencies and modes can be calculated independent of the total oscillation, in both cases 'sliding' $2 N$, respectively. Altogether, they form general solutions for all partial motions. At each switching time the integration constants must be adjusted to the initial conditions (7) and (12), respectively. The only numerical task in this procedure is to determine all roots of the characteristic equation for the natural frequencies. This, however, can be achieved with high accuracy using known methods. The way of calculation for distinct numbers $N$ will not be given in detail. The system matrices (4) for different numbers of elements will also not be presented explicitly. Both of them count among the elementary problems of the Finite Element method. Thus, the essential point is that the three linear partial problems can be calculated analytically. The nonlinear problem of integrating the total oscillation thus concentrates on the determination of the switching 
times on the basis of the switching conditions (9) and (16). This is the inherent numerical problem. As the conditions (9) and (16) cannot be strictly fulfilled, they are substituted by approximations. The time axis is passed in constant steps $\Delta t$. Due to the known analytical solutions in each partial motion, the functions $\dot{y}(t)$ and $R(t)$ can be calculated exactly in two successive times $t$ and $t+\Delta t$. The remaining point of interest is to know whether the interval $(t, t+\Delta t)$ contains a switching time. This is checked by examining

$$
\begin{aligned}
& \operatorname{sgn}(\dot{y}(t+\Delta t))=-\operatorname{sgn}(\dot{y}(t)), \\
& \operatorname{sgn}\left(R_{0}-|R(t+\Delta t)|\right)=-\operatorname{sgn}\left(R_{0}-|R(t)|\right) .
\end{aligned}
$$

If one of the conditions is met, $t_{i}$ is approximately equated to $t+\Delta t$. The switching time within the obtained time step $\Delta t$ can be determined more exactly by an iteration, e.g. by the method of bisection. The optimum choice of a time step for a given problem is not known. The fundamental presumption is to assume that the time step $\Delta t$ includes only one switching time. This condition cannot be safely checked. Switching times may be skipped or-if the time step $\Delta t$ contains several ones-be chosen wrongly. In general, this has fatal consequences for the solution (see [7,8]). From the pragmatic point of view, $\Delta t$ can be determined by means of numerical tests. As to the problem under consideration, $\Delta t$ depends on the number $N$ of the finite elements, the force law and the sequence of sticking and sliding states, i.e. on the properties of the mechanical system and the excitation.

\section{The parameters of the problem}

In order to calculate realistic oscillations, certain parameters have to be chosen. They follow the mechanical properties of a real model used for experimental investigations on the subject [9]. For the excitation a constant force amplitude $F_{0}=2.4 \mathrm{~N}$ is chosen. Geometry $(l=2.47 \mathrm{~m})$, stiffness $\left(E I=1.09 \times 10^{4} \mathrm{Nm}^{2}\right)$ and mass properties $(35.4 \mathrm{~kg} / \mathrm{m})$ of the beam are taken into account by the lowest natural angular frequency $\omega_{\mathrm{g} 1}=10.1 \mathrm{~s}$ in the state 'sliding'. The displacement $y$ is referred to the deflection $y_{s g}=0.34 \mathrm{~mm}$ of a clamped-free beam loaded by a static force $F_{0}$. The reference quantity for the velocity $\dot{y}$ is $\dot{y}_{s g}=y_{s g} \omega_{g \mid}$. Experimental investigations [9] yielded for friction between steel and plastics (increasing characteristic) $d=30 \mathrm{Ns} / \mathrm{m}$, for friction between steel and brass (falling characteristic) $d=-30 \mathrm{Ns} / \mathrm{m}$. For the Rayleigh damping $\alpha=2.5 \times 10^{-2} / \mathrm{s}$ and $\beta=5.5 \times 10^{-5} \mathrm{~s}$ are chosen. The lowest natural mode in the case 'sliding' then has as dimensionless damping $D_{g 1}=0.0015$ which is realistic for steel beams. The damping of all higher modes of all partial states grows almost linearly as the order of the natural modes increases (Table 1).

The control parameters of the problem are the relative exciting frequency $\Omega / \omega_{g 1}$ and the relative threshold for sliding $R_{0} / F_{0}$. With the dimensionless time $\tau=\Omega t / 2 \pi$ one obtains the dimensionless excitation period $T=1$. Thus, the time step $\Delta \tau$ can be chosen as a pure number from now. Due to the modal representation of the linear partial problems, the analytical solutions are composed of $2 N$ or, respectively, $2 N-1$ harmonic oscillations with an equivalent number of natural frequencies. Thus, the frequency spectrum mainly depends on the involved number $N$ of finite elements. The lowest and the highest frequencies are compared in Table 2.

With regard to smooth beam dynamics, FE discretizations yield good results for the lowest frequencies $\omega_{g 1}$ and $\omega_{h 1}$ even for small $N$. As the number of the higher modes increases, the accuracy decreases considerably. The lower ones can be improved by choosing a larger $N$. However, it has to be taken into account that the additional higher frequencies also have a high error rate. For example, calculating the frequency $\omega_{g 20}$ according to the continuum theory one obtains the exact value $\omega_{\mathrm{g} 20}=12800 \mathrm{~s}$ instead of the frequency given in Table 2 . Furthermore, the numerics may be complicated by the fact that the values of lowest and highest frequency differ by orders of magnitude. Thus, treating non-smooth dynamical problems, emerging from finite element

Table 1

Damping coefficients of the lowest and highest natural modes of the linear partial problems for $N=10$

\begin{tabular}{ll}
\hline State 'sliding' & State 'sticking' \\
\hline$D_{k 1}=0.0015$ & $D_{h 1}=0.0015$ \\
$D_{k 21}=0.47$ & $D_{l 11}=0.39$ \\
\hline
\end{tabular}


Table 2

Lowest and highest natural frequencies of the linear partial problems for different numbers of elements

\begin{tabular}{lll}
\hline Number of elements & State 'sliding' & State 'sticking' \\
\hline 2 & $\omega_{g 1}=10.1 \mathrm{~s}$ & $\omega_{h 1}=44.8 \mathrm{~s}$ \\
$\omega_{g 4}=629 \mathrm{~s}$ & $\omega_{h 3}=448 \mathrm{~s}$ \\
10 & $\omega_{g 1}=10.1 \mathrm{~s}$ & $\omega_{h 1}=44.4 \mathrm{~s}$ \\
& $\omega_{g 4}=349 \mathrm{~s}$ & $\omega_{h 3}=301 \mathrm{~s}$ \\
& $\omega_{g 20}=17300 \mathrm{~s}$ & $\omega_{h 1}=14300 \mathrm{~s}$ \\
\hline
\end{tabular}

discretizations, one has to face the fact that on the one hand, all natural modes are necessary for the calculation, but on the other hand, the higher ones are very inaccurate.

\section{The influence of the force law on periodic solutions}

A harmonic excitation is expected to cause periodic responses. These stationary responses are calculated from transient oscillations. First, the influence of the relative threshold $R_{0} / F_{0}$ on the type of oscillation is considered. In the ideal case, i.e. Coulomb's law disregarding internal damping, Fig. 3 shows orbits for the coordinate $y$ of the friction device. The excitation $\Omega=2.45 \omega_{g 1}$ is approximately in the middle between both lowest resonances of the two linear partial systems. The number of elements is $N=2$. The time step for the determination of the switching times is $\Delta \tau=0.5 \times 10^{-3}$. The exact localization of the switching times is made by iteration.

Because in a state 'sticking' the displacement is constant and the velocity zero, the whole state is indicated by one point on the $y$-axis in the phase plane. For a better understanding the letters in Fig. 3 designate the sequence of the partial motions during one stationary response period. The meanings are: $H=$ 'sticking', $G^{+}$'sliding' with $\dot{y}>0, G^{-}$'sliding' with $\dot{y}<0$. As the threshold $R_{0}$ decreases, the amplitude of the response increases under the same excitation. The number of sticking states in one period reduces. The oscillations are unique. Bifurcations do not occur. The stationary response is independent of the initial conditions and the initial state at the time $\tau=0$.

Under the same presumptions Fig. 4 shows the stationary oscillations for a decreasing characteristic.

The solution is no longer unique. There exist two different dynamic equilibrium positions, which belong to different initial conditions. Both solutions are symmetric to each other and show the same sequence of partial states. As the threshold $R_{0}$ decreases, bifurcations (subharmonic solutions) occur. The amplitudes increase considerably and the number of sticking states grows.

In order to compare the influence of Coulomb's law with those of an increasing characteristic on the type of oscillation a discretization with $N=10$ is made. It is sufficient to consider exemplarily the arbitrarily chosen
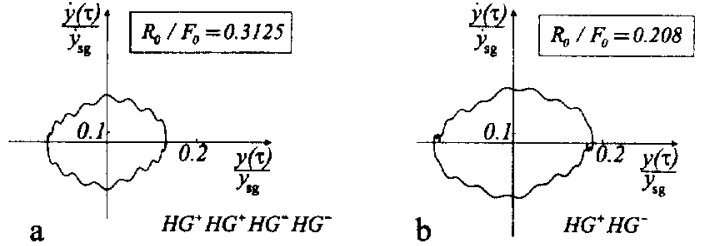

Fig. 3. Orbits in the case of Coulomb's law for the motion of the friction device. (a) $R_{0}$ large; (b) $R_{0}$ small.
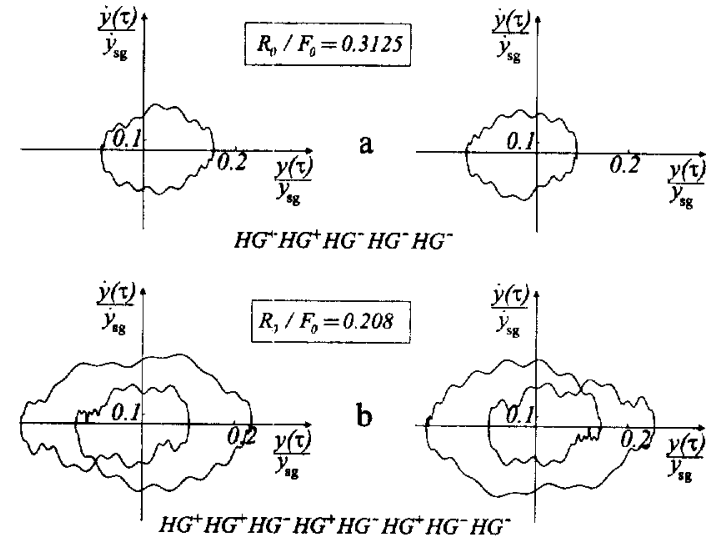

Fig. 4. Orbits in the case of a decreasing characteristic for the motion of the friction device. (a) $R_{0}$ large; (b) $R_{0}$ small. 

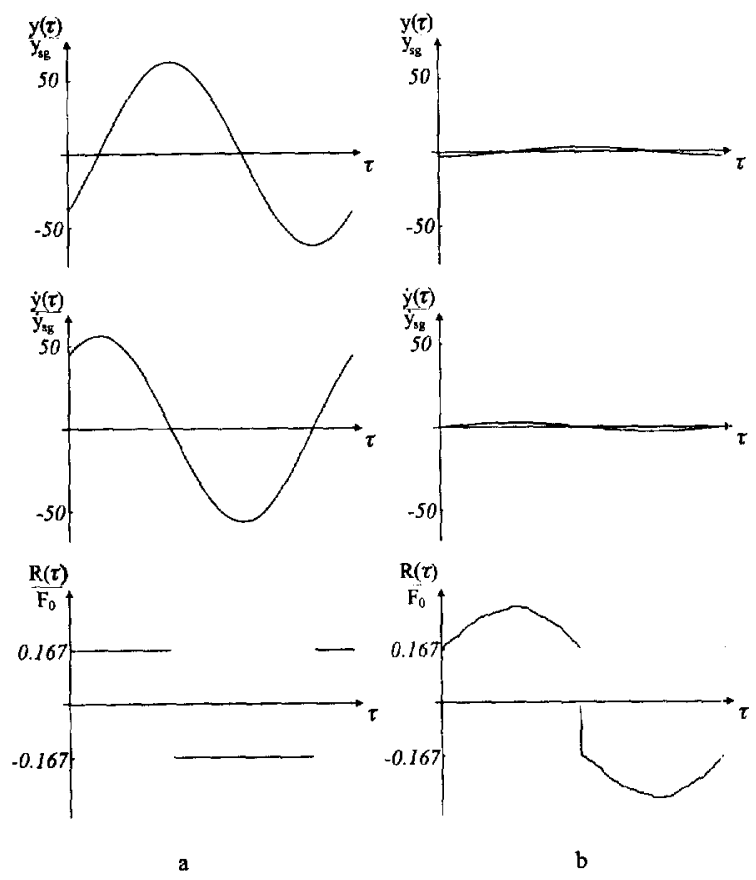

Fig. 5. Exemplary stationary period of coordinate, velocity and contact force. (a) Coulomb; (b) increasing characteristic.

value $R_{0} / F_{0}=0.167$, since both force laws always yield unique and nonbifurcated solutions. The chosen time step is $\Delta \tau=2 \times 10^{-3}$ with iteration. Internal damping is neglected. The excitation frequency $\Omega=0.99 \omega_{g 1}$ is close to the resonance of the partial state 'sliding'. Fig. 5 shows coordinate $y$, velocity $\dot{y}$ and contact force $R$ versus time during one stationary period of the response.

Taking transient motions into consideration, the transient oscillation under Coulomb's law has a duration of about 300 excitation periods, for the increasing characteristic the stationary oscillation is reached already after 20 periods. This impressive influence of additional dissipation also becomes evident in the results shown in Fig. 5. In the case of the increasing characteristic, the amplitudes of coordinate and velocity are much lower compared to those of Coulomb's law. Furthermore, it is remarkable that under Coulomb's law the chosen excitation frequency does not cause sticking, but only sliding in different directions. In the case of the increasing characteristic short states of sticking occur.

\section{The influence of the number of elements and the viscous internal damping}

So far, the numerical efforts depending on the number $N$ of the employed elements were not discussed and viscous internal damping was not considered. Fig. 6 shows three different cases for the same values $\Omega=1.1 \omega_{g 1}$, $R_{0} / F_{0}=0.275$. The figures show one period of the stationary response of coordinate, velocity and contact force versus time after transient oscillations of 20 excitation periods, Coulomb's law being valid in all cases.

Fig. 6(a) and (b) allow a comparison of the results for $N=2$ and $N=10$ without damping. Qualitatively, the course of all curves is the same in both cases, but the values of the amplitudes differ significantly. Obviously, the small number $N=2$ of elements is not sufficient to obtain a quantitatively correct result. The principal numerical problem becomes evident in the course of the contact force. For $N=2$ it is comparatively smooth. For $N=10$ the transitions between long lasting sticking and sliding processes are characterized by rapid changeovers because switching from one state to another occurs in short time intervals. During one period a total of 12 switching times for $N=2$ and 60 for $N=10$ exist. This also explains the extremely different necessary time steps $\Delta \tau=5 \times 10^{-3}$ for $N=2$ and $\Delta \tau=5 \times 10^{-5}$ for $N=10$. They are caused by the numerous inaccurate higher modes of the linear partial motions mentioned in Section 4, which are mainly apparent near switching points. 

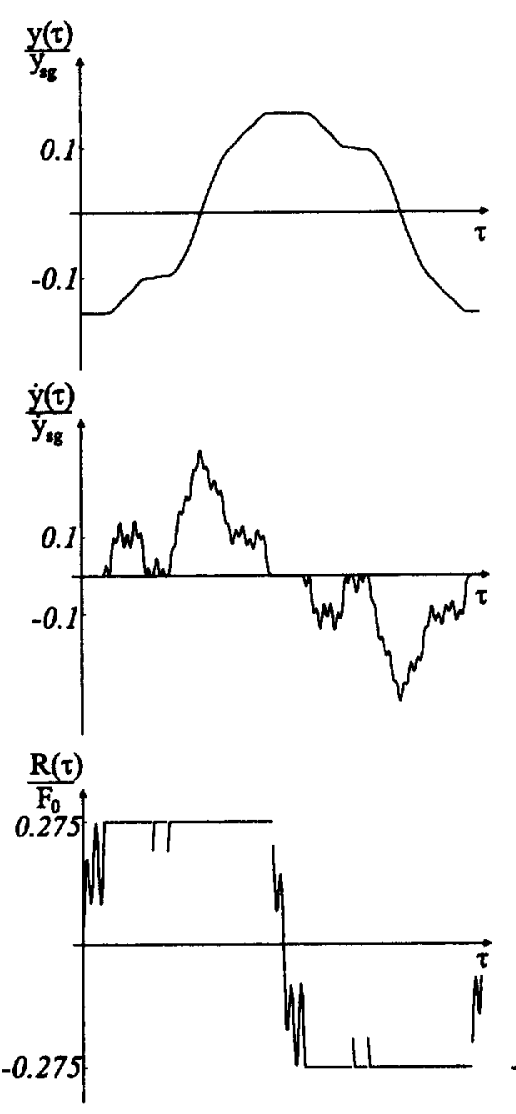

a
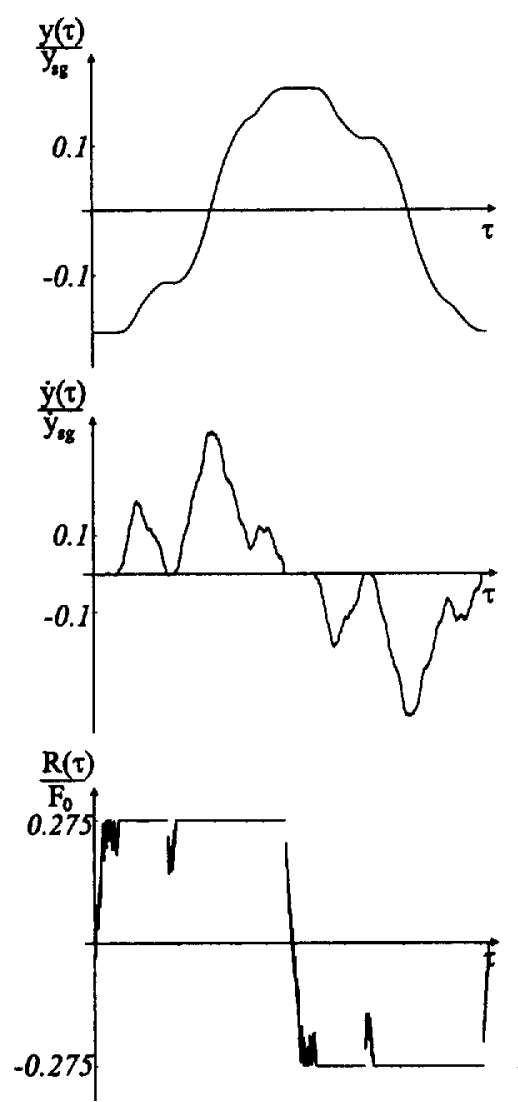

b
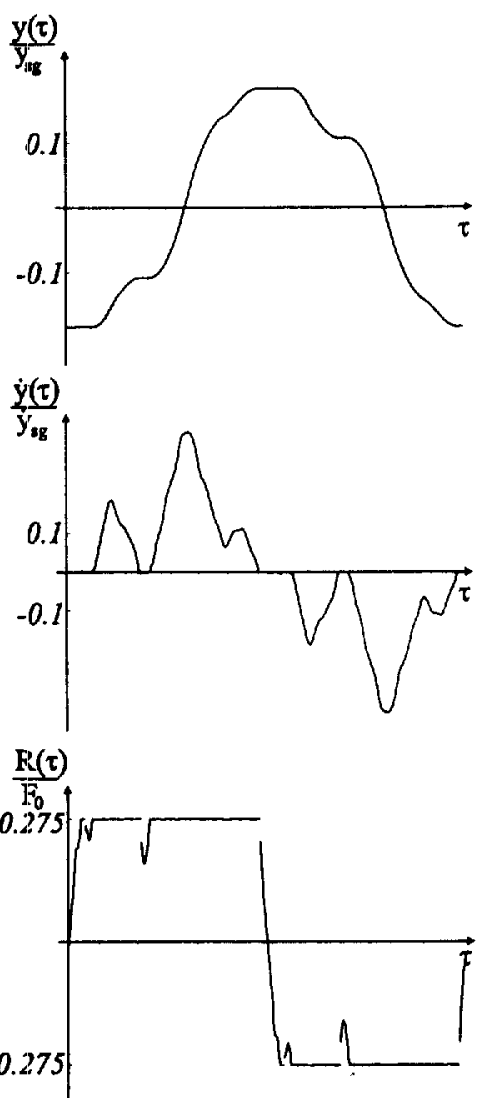

c

Fig. 6. Stationary response of coordinate, velocity and contact force. (a) $N=2$, no damping; (b) $N=10$, no damping; (c) $N=10$, Rayleigh damping.

On the one hand, an exact result requires a sufficiently large number of finite elements to capture the lower modes exactly, on the other hand the consideration of all inaccurate higher modes causes a great computational effort which is not justifiable for practical interests. This conflict is solved by taking account of internal damping in the beam. In the case of Rayleigh damping, the basic modes decisive for the actual oscillation are slightly damped, all higher modes are strongly damped (see Table 1). Above all, the higher modes lose their influence when determining the switching times. Additionally, the calculation becomes less sensitive with regard to numerical inaccuracies resulting from the calculation of the switching points. This is shown in Fig. 6(c). It is particularly remarkable that the course of the contact force versus time becomes smooth. In spite of $N=10$, the number of switching times in one period reduces to 12 . The necessary time step is only $\Delta \tau=2.5 \times 10^{-2}$, i.e. it is by almost one order of magnitude larger than in the case $N=2$ without damping. The factor by which the computational effort is reduced compared to the results of Figs. 6(b) and (c) is approximately 300.

\section{Conclusion}

The FE-formulation of a dynamic contact problem is considered. Non-smooth oscillations due to dry friction exist. A distinct separation of states of sticking and sliding in a friction device leads to a sequence of different linear partial motions, which is not known a priori because it depends on time history. Classical treatments of contact problems with FEM turn over non-smooth motions into smooth problems by regularisation of the friction law. This is achieved by introducing an elastic contact stiffness at the point of contact. The general consequences of this heuristic procedure have already been discussed in [10]. As far as the considered example 
is concerned, an elastic contact element would give rise to a change of the natural frequencies of the partial linear states of the mechanical system. Mostly, the higher modes would be affected. But these are responsible for the numerical problems which have been discussed. Much more severe is the fact, that states of 'sticking', which can be observed in realistic experiments [9], are eliminated by regularisation methods.

In contrast, the numerical effort for the non-smooth problem considered is reduced considerably by introducing internal damping in the beam itself. It influences mostly all higher modes of the linear partial motions, describing sticking and sliding at the friction device separately. The influence of all higher modes with less accuracy due to FEM vanishes. The contact force is smoothened realistically while the distinct separation of all properties of the friction device still exists.

\section{References}

[1] H. Wissbrock, Untersuchungen zur Fugendämpfung zusammengesetzter Bauteile (VDI Verlag, Düsseldorf, 1985).

[2] S. Dimova, K. Meskouris and W.B. Krätzig, Numerical technique for dynamical analysis of structures with friction device, Earthquake Engrg. Struct. Dyn. 24 (1995) 881-898.

[3] P. Vielsack, Modellbildung und numerische Probleme bei Reibschwingern mit Pausen, ZAMM 78 (1998) S2, 785-786.

[4] J.J. Moreau and P.D. Panagiotopoulos, eds., Nonsmooth Mechanics and Applications, CISM, Vol. 302 (Springer Verlag, Wien, 1988).

[5] J.P. Meijard, Efficient numerical integration of the equation of motion of non-smooth mechanical systems, ZAMM 77(6) (1997) 419-427.

[6] W.T. Thompson, Theory of Vibrations (Unwin Hyman Ltd., London-Sidney-Wellington, 1988).

[7] P. Vielsack and A. Hartung, An example for the Orbital Stability of permanently disturbed Non-smooth Motions, ZAMM, to appear.

[8] P. Vielsack, Orbitale Stabilität nichtglatter Bewegungen bei permanenten numerischen Störungen, ZAMM 79 (1999) S1, $104-108$.

[9] H. Kammerer, Nichtglatte Dynamik eines fremderregten elastischen Balkens mit Reibglied, Diss. Universität Karlsruhe, 1998.

[10] P. Vielsack, Regularisierung des Haftzustandes bei Coulombscher Reibung, ZAMM 76(8) (1996) 439-446. 